\title{
Optimal Control for a Tuberculosis Model with Undetected Cases in Cameroon
}

\author{
D. P. Moualeu ${ }^{\text {a }}$, M. Weiser ${ }^{b, c}$, R. Ehrig ${ }^{\mathrm{a}}$, and P. Deuflhard ${ }^{\mathrm{a}, \mathrm{b}, \mathrm{c}, \mathrm{d}}$ \\ ${ }^{a}$ Computational Systems Biology Group, Zuse Institute Berlin (ZIB), Takustr. 7, 14195 \\ Berlin, Germany \\ ${ }^{b}$ Research Group Computational Medicine, Zuse Institute Berlin (ZIB), Takustr. 7, 14195 \\ Berlin, Germany \\ c DFG Research Center Matheon "Mathematics for Key Technologies", Berlin, Germany \\ ${ }^{d}$ Department of Mathematics and Computer Science, Freie Universität Berlin, Arnimallee \\ 6, 14195 Berlin, Germany
}

\begin{abstract}
This paper considers the optimal control of tuberculosis through education, diagnosis campaign and chemoprophylaxis of latently infected. A mathematical model which includes important components such as undiagnosed infectious, diagnosed infectious, latently infected and lost-sight infectious is formulated. The model combines a frequency dependent and a density dependent force of infection for TB transmission. Through optimal control theory and numerical simulations, a cost-effective balance of two different intervention methods is obtained. Seeking to minimize the amount of money the government spends when tuberculosis remain endemic in the Cameroonian population, Pontryagin's maximum principle is used to characterize the optimal control. The optimality system is derived and solved numerically using the forward-backward sweep method (FBSM). Results provide a framework for designing cost-effective strategies for diseases with multiple intervention methods. It comes out that combining chemoprophylaxis and education, the burden of TB can be reduced by $80 \%$ in 10 years
\end{abstract}

Keywords: Tuberculosis, Optimal control, Diagnosis Campaign, Nonlinear dynamical systems.

2000 MSC: Primary: 34D05, 92B05, 34D35, 65L09, 37G10, Secondary:34D23

\section{Introduction}

Tuberculosis (TB) is a preventable and curable disease caused by Mycobacterium tuberculosis (Mtb) that most often affects the lungs. To date, TB claims the second largest number of victims due to a single infectious agent right after

\footnotetext{
Whasponding author, email: moualeu@zib.de, tel: +49-30-84185-355, fax: +49-3084185- 125 .
}

Preprint submitted to Communication in Nonlinear Science and Numerical SimulationMay 6, 2014 
Human Immunodeficiency Virus and Acquired Immune Deficiency Syndrome (HIV/AIDS) [39]. According to the WHO data published in April 2011 the TB case detection rate (all forms) in Cameroon was last reported at $69 \%$ in 2010 and the TB deaths reached 3,647 or $1.54 \%$ of total deaths. The age adjusted death rate of 21.89 per 100,000 of population ranks Cameroon 68th in the world [39].

Adult mortality has a significant effect on national economies, through both the direct loss of productivity among those of working age and by altering fertility, incentives for risk-taking behavior, and investment in human and physical capital [24]. TB is the most important cause of adult death due to infectious disease after HIV/AIDS. TB has its greatest impact on adults between the ages of 15 and 59 [38]. Therefore most economically productive persons in society, parents on whom development and survival of children depend, are affected. TB places an extraordinary burden on those afflicted by the disease, their families, communities and on government budgets. In fact, the greatest burden of TB falls on productive adults who, once infected, are weakened and often unable to work. The burden of taking care of sick individuals usually falls to other family members and, besides putting them at greater risk of infection, can lower their productivity [24]. Diagnosed individuals with TB are often medically quarantined for a period of time, which can affect their financial well-being. The infected population has an economic impact on their families and in turn their countries' national economies through their inability to contribute financially, as they are often unable to be productive workers. Along with loss of productivity, the TB treatment charge can be for the Government significant. Average household spending on TB can account for as much as $8-20$ percent of annual household income, varying by region $[13,35]$.

Some TB models in the literature have discussed control of the disease by looking at the role of disease transmission parameters for time dependent control strategies. Time dependent control strategies have been applied for the studies of HIV models [15, 22], two strain tuberculosis models [21], a TB model with lost sight class to reduce the rate by which people become lost sight [14], and a SARS model with quarantine [41]. Some authors [19, 33] discussed the optimal control on a model with reinfection. Time dependent optimal controls on chemoprophylaxis of both diagnosed and latently infected individual, and disease relapses are considered in [1] to reduce the actively infected individual populations. In their recent article, Silva and Torres [37] studied the time optimal control of TB in Angola using a model with early and persistent latency. Approaches of studying control strategies produced valuable theoretical results which suggest or design epidemic control programs. However, none of the previous studies considered the key combined role of undiagnosed infectious population and quiting of the treatment on TB propagation. The goal in this paper is to minimize the number of people who are undiagnosed infectious, lost sight, thus also the number of people who die due to the infection. The number of TB new-cases, even if the success rate of treatment remains the same will decrease implicitly. In this paper, we compute an optimal way to minimize the cost of $\mathrm{TB}$ on a calibrated model taking into account the Cameroonian last 
trend of the diseases and resulting parameters. The TB cost here means the expenses to fight TB and save human life.

Two control strategies are considered. The first consists of education of the population about TB and large scale diagnosis campaigns. Through education, one can decrease the number of undiagnosed infectious people inside the population. Using the combined effect of education and free of charge treatment, one can first reduce the number of people infected in the population, make return people who have quitted the treatment to the hospital and accelerate the detection of newly infected people who would stay to be treated naturally or through self-medication and traditional medicine. A proportion of them will die without appropriate treatment. According to FAO, less than $73 \%$ of the Cameroonian population goes to the hospital after first disease symptoms. Because of the inaccessibility of certain regions of the country, access to health facilities is often difficult. Besides treatment facilities, some rural and even urban population prefer sometimes to use traditional medicine or self-medication for which the efficiency has not been established yet for TB. An increase in the treatment access should help to reduce the lost sight and undiagnosed classes. The immediate consequence will be a reduction in the number of infectious and then, on the number of diagnosed infectious.

The second TB control approach is the chemoprophylaxis treatment. According to the National Committee of Fight against TB (NCFT), the chemoprophylaxis of latently infected population is currently not practiced in Cameroon. The number of latently infected individuals that may develop an active TB will decrease if the chemoprophylaxis is practiced. The impact of both strategies on TB dynamics will be discussed. We intend to determine optimal control strategies that minimize not only undiagnosed infectious but also lost-sight and diagnosed infectious individuals which are the source of TB spread. The optimal controls are completely characterized and optimal solutions are depicted.

\section{Modeling intervention methods}

Mycobacterium tuberculosis (Mtb) spreads through the air from an infectious person to a susceptible person. Two kinds of tests are used to determine if a person has been infected with TB bacteria: the tuberculin skin test and TB blood analysis. A positive TB skin test or TB blood test only tells that a person has been infected with TB bacteria and does not tell whether the person has latent TB infection or has progressed to TB disease [12]. Other tests, such as a chest X-ray and a sample of sputum, are needed to see whether the person has TB disease. Some TB treatment and prevention options are carried out in some rural and urban hospitals in Cameroon. However, the treatment of mild infections, classified as latent infections in our model, is not effective in Cameroon. On the other hand, infective individuals classed as infectious in our model, require a hard treatment of six months in the hospital. As preventive measures, population can be diagnosed, and latent TB infections can be treated to reduce the bacterial load in their body. This last approach effectively reduces the risk that TB infection will progress to TB disease. Certain groups are at 
very high risk of developing TB disease once infected. Every effort should be made to begin appropriate treatment and to ensure completion of the entire course of treatment for latent TB infection [12].

A finite (non-constant) total population at time $t$, denoted by $N(t)$, is subdivided into the following mutually exclusive sub-populations:

$\mathrm{S}$ susceptible : healthy people not yet exposed to TB

E latently infected: exposed to TB but not infectious

I diagnosed infectious: have active TB confirmed after a sputum examination in a hospital

J undiagnosed infectious: have not yet been to a hospital for diagnosis but are active for confirmation by a sputum examination

L lost sight: people who have been diagnosed as having active TB, begun their treatment and quitted before the end

$\mathrm{R}$ recovered: people cured after treatment in the hospital

In Africa, reliable TB tests [23] are often missing or too expensive. Hence, TB diagnosis based on a single sputum examination can often only be classified as "probable" or "presumed", and cannot detect cases of less infectious forms of TB [40]. Therefore, the model is based on the following assumptions, established from behaviours of people in different epidemiological classes.

1. Mtb transmission from diagnosed infectious to susceptible population, due to education on the infection is limited. it is therefore modeled using a standard mass action or frequency-dependent force of infection.

2. Mtb transmission from undiagnosed infectious to susceptible population, due to their level of education on the disease is modeled by a densitydependent force of infection.

These arguments abide on the fact that diagnosed infectious people are in most cases hospitalized for at least 2 months or are advised to lessen their infectiousness in their residing neighbourhood. Their distribution in the population is not necessarily homogeneous. Since undiagnosed infectious remain inside the population, there is an unlimited possibility of contacts with the susceptible population [3]. We therefore assume a density dependent force of infection for hospital inmates [4].

All recruitment is into the susceptible class and occurs at an average scale $\Lambda$. The fixed rate of non-disease related death is $\mu$, thus $1 / \mu$ is the average lifetime. Susceptible individuals acquire TB infection from individuals with active TB and lost sight at a rate $\nu(I, J, L)$ is given by

$$
\nu(I, J, L)=\beta_{1} \frac{I}{N}+\beta_{2} \frac{L}{N}+\beta_{3} J,
$$

where $\beta_{i}, i=1,2,3$, are the effective contact rates of diagnosed, lost sight and undiagnosed infectious sufficient to transmit infection to susceptible people. The 
effective contact rates $\beta_{i}$ in a given population for tuberculosis are measured in effective contacts per unit time. This may be expressed as the product of the total contact rate per unit time $\left(\eta_{i}\right)$ by the risk of infection $\left(\phi_{i}\right)$ given contact between an infectious and a susceptible individual, $\beta_{i}=\eta_{i} \phi_{i}$. This risk is called the transmission risk. A proportion $p$ of the latently-infected individuals develop fast active TB and the remainder $(1-p)$ develop latent TB and enter the latent class $E$. Among latently-infected individuals developing active TB, a fraction $f$ is assumed to undergo a fast progression directly to the diagnosed infectious class $I$, while the remainder $(1-f)$ enters the undiagnosed infectious class $J$. We set

$$
p_{1}=p f \quad \text { and } \quad p_{2}=p(1-f) .
$$

Once latently infected with Mtb, an individual will remain so for life unless reactivation occurs. Latently infected individuals are assumed to acquire some immunity as a result of infection, which reduces the risk of subsequent infection but does not fully prevent it.

Due to endogenous reactivation, a fraction $1-r_{1}$ of latently infected individuals who did not receive effective chemoprophylaxis become infectious with a constant rate $k$, and reinfect after effective contact with individuals in the active TB classes or lost sight at a rate

$$
\lambda_{e}=\sigma_{1} \nu(I, J, L)
$$

where $\sigma_{1}$ is the factor reducing the risk of infection as a result of acquiring immunity for latently infected individuals. Among latently infected individuals who become infectious, the fraction $h$ is diagnosed and treated under the "Stop TB" program, while the remaining $1-h$ is not diagnosed and becomes undiagnosed infectious $J$. We assume that after some time suffering from TB, some undiagnosed infectious decide to go to hospital with a rate $\theta$. Also, we assume that among diagnosed infectious who had begun their treatment therapy, a fraction $r_{2}$ of $I$ has taken all the dose and has made all the sputum examinations and will be declared cured from the disease. Some diagnosed infectious who have not finished their dose of drugs and sputum examinations or whose treatment was unsuccessful, will not return to the hospital for the rest of sputum examinations and check-up. They will enter the class of lost sight $L$ at a constant rate $\alpha$. Lost sight can return to the hospital at a constant rate $\delta$. As suggested by Murray et al. [29], recovered individuals can only have partial immunity. Hence, they can undergo a TB reactivation or relapse with a constant rate $\gamma$. The remainder can be reinfected (exogenously) after an effective contact with individuals in the active TB classes and lost sight at a rate

$$
\lambda_{r}=\sigma_{2} \nu(I, J, L)
$$

where $\sigma_{2}$ is the factor reducing the risk of infection as a result of acquiring partial immunity for recovered individuals. Due to their own immunity, traditional medicine, natural recovery and drugs bought in the street (practised in sub-Saharan Africa), a fraction of lost sight and undiagnosed infectious can 
spontaneously recover at constant rates $\rho$ and $\omega$, respectively and enter the latent class $E$ and recovery class $R$ respectively. A description of the parameters is summarized in Table 1.

Controls are represented as functions of time and assigned reasonable upper and lower bounds. In [27, 28], it has been shown that undiagnosed infectious population plays a fundamental role on TB propagation through sensitivity analysis. Therefore, the optimal control strategies aim to minimize the number of undiagnosed infectious and lost sight in the population. First, $u(t)$ represents the effort on education which allows people to go to the hospital, to be diagnosed early and treated from the disease (to reduce the number of individuals that may be undiagnosed infectious). Second, $v(t)$ measures the rate of treatment of TB latent infections in each time period. The whole model flow diagram with

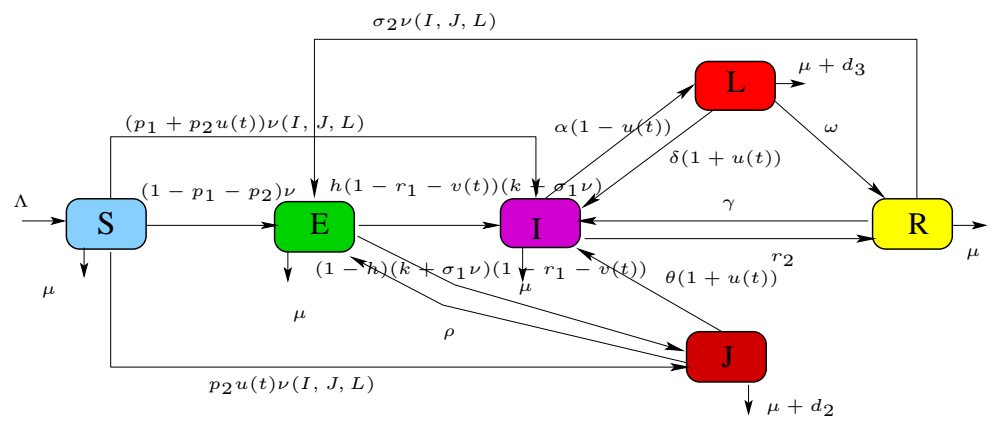

Figure 1: Transfer diagram for a transmission dynamics of tuberculosis.

control is shown in Figure 1, and given as ODE system (2) below.

$$
\left\{\begin{aligned}
\dot{S} & =\Lambda-\nu(I, J, L) S-\mu S, \\
\dot{E} & =\left(1-p_{1}-p_{2}\right) \nu(I, J, L) S+\rho J+\sigma_{2} \nu(I, J, L) R \\
& -\sigma_{1}\left(1-r_{1}-v(t)\right) \nu(I, J, L) E-A_{1} E \\
\dot{I} & =\left(p_{1}+p_{2} u(t)\right) \nu(I, J, L) S+(1+u(t)) \delta L+(1+u(t)) \theta J+\gamma R \\
& +h\left(1-r_{1}-v(t)\right)\left(k+\sigma_{1} \nu(I, J, L)\right) E-A_{2}(t) I, \\
\dot{J} & =p_{2}(1-u(t)) \nu(I, J, L) S+(1-h)\left(1-r_{1}-v(t)\right)\left(k+\sigma_{1} \nu(I, J, L)\right) E-A_{3} J, \\
\dot{L} & =\alpha(1-u(t)) I-A_{4} L, \\
\dot{R} & =r_{2} I+\omega L-\sigma_{2} \nu(I, J, L) R-A_{5} R,
\end{aligned}\right.
$$

with

$$
\begin{aligned}
& A_{1}(t)=\mu+k\left(1-r_{1}-v(t)\right), \quad A_{2}=\mu+d_{1}+r_{2}+\alpha(1-u(t)), \\
& A_{3}(t)=\mu+d_{2}+\theta(1+u(t))+\rho, \quad A_{4}=\mu+d_{3}+\delta(1+u(t)) \text { and } A_{5}=\gamma+\mu,
\end{aligned}
$$

subject to the initial conditions

$$
S(0)=S_{0}, \quad E(0)=E_{0}, \quad I(0)=I_{0}, \quad J(0)=J_{0}, \quad L(0)=L_{0} \quad R(0)=R_{0} .
$$

Table 1 presents parameter values and descriptions. The control functions $u(t)$ and $v(t)$ have to be bounded and Lebesgue integrable functions. $u(t)$ represents 
supplementary time dependent efforts of education campaigns, applied during a time interval $[0, T]$ to the whole population. The control function $v(t)$ measures the time dependent efforts on the preventive treatment of latently infected individuals to reduce the number of individuals that may be infectious. This control will have an impact on the output flow of people from the latently infected class to infectious classes. The coefficient $1-u(t)$ is a decreasing factor for fast route flow to undiagnosed population as result of education. This factor aims to reduce the number of people becoming fast undiagnosed infectious and developing cavitation. The coefficient $v(t)$ represents the effort that prevents the inflow to the undiagnosed infectious classes to reduce the number of undiagnosed individuals developing cavitation. Our control problem involves the number of individuals with latent and active tuberculosis infections and the cost of applying chemoprophylaxis education and treatment controls $u(t)$ and $v(t)$ to be minimized subject to the differential equation (2). A description and the estimated values of the parameters is summarized in Table 1 such that the model fits with TB dynamics in Cameroon from $1994-2013$.

Note that in equation (2) the control $u(t)$ moves infectious individuals from classes $J$ and $L$ to class $I$ and decreases the evolution to $J$ and $L$ classes. However, the control $v(t)$ reduces the progression rate from the latently infected class to the infectious classes. Since treatment effectively decreases the number of infectious individuals in the population, the control functions may provide a model of the impact of education and chemoprophylaxis of both latent and active infection.

\section{Comparison to measurement data}

Numerical simulations of model system (2) are carried out using a linearly implicit Euler scheme in Matlab, version R2009. The total population of Cameroon in 1994 is given by $N=13,240,337$ [31]. Using measurable data, model (2) gives a very good fit to the Cameroonian data for the period 1994-2010 [39], as depicted in Figure 2. Estimated transmission parameters are performed using a state of the art Gauss-Newton Method as presented in [27, 28] and the deterministic model appears to capture all the qualitative properties of the observed pattern. Hence, model (2) can be used to gain realistic insight into tuberculosis transmission dynamics at least for a limited period.

\section{Modeling the optimal control problem}

The goal is to solve the following problem: given initial population sizes of all six classes, $S_{0}, E_{0}, I_{0}, J_{0}, L_{0}$ and $R_{0}$ find the best strategy in terms of efforts of education or chemoprophylaxis or both, that would minimize the number of people who die from the infection while at the same time minimizing the cost of the strategy. There are various ways of expressing such a goal mathematically. 


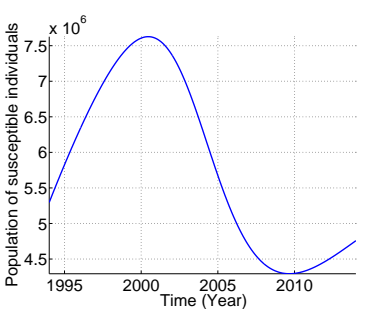

(a) $\mathrm{S}$

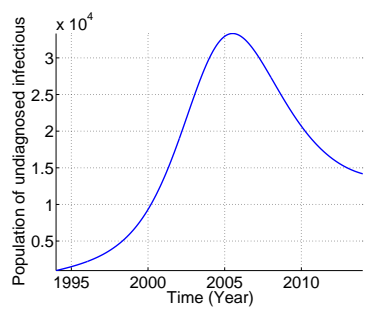

(d) $\mathrm{J}$

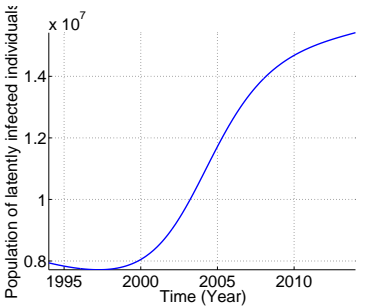

(b) $\mathrm{E}$

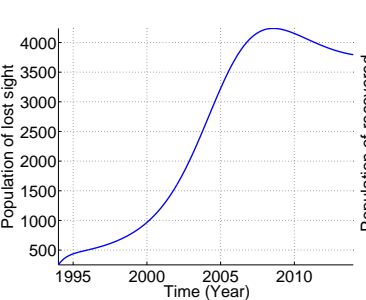

(e) $\mathrm{L}$

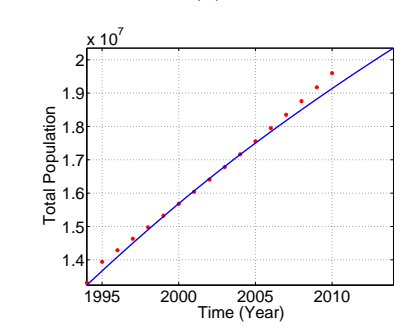

(g) $\mathrm{N}$

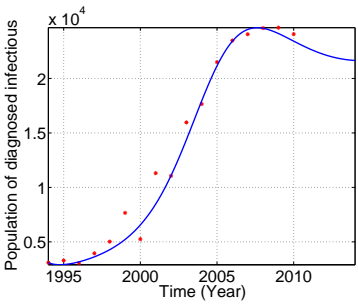

(c) I

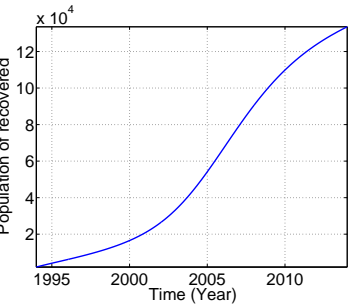

(f) $\mathrm{R}$

Figure 2: Evolution of model (2) showing the estimated state trajectories of susceptible, latently infected, diagnosed infectious, undiagnosed infectious, lost sight, recovered and total population classes. The dot plots represent the year-by-year trend and variability in yearly case reports over the period 1994-2010.

\section{Formulation}

The total cost on a finite time horizon $[0, T]$ consists of the cost induced by the disease itself and the cost induced by education and chemoprophylaxis efforts. We split the disease costs into costs induced by death due to TB,

$$
\int_{0}^{T} B_{1}\left(d_{1} I(t)+d_{2} J(t)+d_{3} L(t)\right) d t,
$$

proportional to the total number of deaths, and those induced by treatment of diagnosed infectious,

$$
\int_{0}^{T} B_{2} I(t) d t
$$

proportional to the number of patients and duration of treatment. Modelling the control cost is a more delicate issue. Linear and quadratic costs are frequently 


\begin{tabular}{|c|c|c|c|}
\hline Parameters & Symbol & Estimate /yr & Source \\
\hline Recruitment rate of susceptible & $\Lambda$ & 679685 & {$[27,31]$} \\
\hline Transmission rate & $\beta_{1}, \beta_{2}$ & 1,4 & {$[27,5]$} \\
\hline Transmission rate & $\beta_{3}$ & $6.05681 \cdot 10^{-06}$ & {$[27]$} \\
\hline Fast route to infectious class & $p_{1}$ & $9.36432 \cdot 10^{-04}$ & {$[27]$} \\
\hline $\begin{array}{l}\text { Fast route to undiagnosed } \\
\text { infectious class }\end{array}$ & $p_{2}$ & $2.43736 \cdot 10^{-02}$ & {$[27]$} \\
\hline $\begin{array}{l}\text { Reinfection parameter of latently } \\
\text { infected individuals }\end{array}$ & $\sigma_{1}$ & $2.38390 \cdot 10^{-04}$ & {$[27]$} \\
\hline $\begin{array}{l}\text { Reinfection parameter } \\
\text { of recovered individuals }\end{array}$ & $\sigma_{2}$ & $0.7 *\left(p_{1}+p_{2}\right)$ & {$[27,2]$} \\
\hline Slow route to active $\mathrm{TB}$ & $k$ & $3.31390 \cdot 10^{-04}$ & {$[27]$} \\
\hline Natural mortality & $\mu$ & $1 / 53.6$ & {$[27,5,31]$} \\
\hline TB mortality of diagnosed infectious & $d_{1}$ & 0.139 & {$[27,5]$} \\
\hline TB mortality of undiagnosed infectious & $d_{2}$ & 0.413 & {$[27]$} \\
\hline TB mortality of lost sight & $d_{3}$ & 0.20 & {$[27]$} \\
\hline $\begin{array}{l}\text { Chemoprophylaxis of latently } \\
\text { infected individuals }\end{array}$ & $r_{1}$ & 0 & {$[27,32]$} \\
\hline Detection rate of active TB & $h$ & 0.828248 & {$[27]$} \\
\hline Recovery rate of diagnosed infectious & $r_{2}$ & 0.758821 & {$[27,32]$} \\
\hline Recovery rate of lost sight & $\omega$ & 0.5 & {$[27]$} \\
\hline Recovery rate of undiagnosed infectious & $\rho$ & 0.131140 & {$[27]$} \\
\hline Relapse of recovered individuals & $\gamma$ & $8.51257 \cdot 10^{-02}$ & {$[27]$} \\
\hline $\begin{array}{l}\text { Diagnosed infectious route } \\
\text { to the lost sight class }\end{array}$ & $\alpha$ & 0.216682 & {$[27]$} \\
\hline $\begin{array}{l}\text { Lost sight route } \\
\text { to the diagnosed infectious class }\end{array}$ & $\delta$ & 0.39 & {$[27]$} \\
\hline Diagnosed rate & $\theta$ & 0.495896 & {$[27]$} \\
\hline
\end{tabular}

Table 1: Estimated numerical values of the TB model parameters as presented in $[27,28]$

considered in the literature $[9,18,20,21,36,30]$, as well as no cost at all but a limitation of resources [6]. As we consider rather long time horizons, limitation of resources is neglected. We impose a control cost

$$
\int_{0}^{T}\left(b_{u}(u)+b_{v}(v)\right) d t
$$

and state qualitative properties of the functions $b_{u}$ and $b_{v}$. As no control induces no cost, we have $b_{u, v}(0)=0$. We assume that the different means of education (TV spots, school education, doctors visiting remote villages etc.) and chemoprophylaxis for latently infected people are employed in an economically optimal way. This implies that improving results, i.e. increasing $u$ or $v$ further, not only leads to additional costs, but does so increasingly, because cheaper means of control are already exploited. Thus, $b_{u, v}$ is monotonely increasing and convex. 
Finally, extending education and chemoprophylaxis to everyone even in the most remote village will lead to unreasonably high cost. This is modelled as barrier property $b_{u, v} \rightarrow \infty$ as $u, v \rightarrow 1$, which also eliminates the necessity of imposing state constraints $u, v \leq 1$.

A plethora of monotone, convex barrier methods is available. Choosing a particular one in a quantiatively well-justified way would require a detailed understanding of the cost and effectivity of several different control means. In lack of such models and data we choose prototypical monotone, convex barrier functions

$$
b_{u}(u)=C_{1} u-C_{2} \log (1-u), \quad b_{v}(v)=C_{1} v-C_{2} \log (1-v)
$$

without further justification.

Finally, optimization over a finite time horizon can be misleading, as the optimizer ignores the sustainability of the situation and hence any cost that might arise after the final time. For this reason, we include an additional final time term, which penalizes the deviation of the population $y_{1}(t)=(I(t), J(t), L(t))$ from the disease-free equilibrium $y^{*}=(0,0,0)$ :

$$
D_{3}\left\|y_{1}(T)-y^{*}\right\|_{2}^{2} .
$$

The overall objective is now

$$
\begin{aligned}
J(u, v) & =\int_{0}^{T}\left\{B_{1}\left(d_{1} I(t)+d_{2} J(t)+d_{3} L(t)\right)+B_{2} I(t)+C_{1} u(t)+D_{1} v(t)\right. \\
& \left.-C_{2} \log (1-u(t))-D_{2} \log (1-v(t))\right\} d t+D_{3}\left\|y_{1}(T)-y^{*}\right\|_{2}^{2} .
\end{aligned}
$$

The set of admissible controls is defined as follows.

$$
\Gamma=\left\{u, v \in L^{1}(0, T) \mid(u(t), v(t)) \in[0,1] \times[0,1] \forall t \in[0, T]\right\}
$$

Thus, we seek an optimal control pair $\left(u^{*}, v^{*}\right)$ such that

$$
J\left(u^{*}, v^{*}\right)=\min _{\Gamma} J(u, v) .
$$

\subsection{Existence of an optimal control solution}

Let us consider an optimal control problem having the form

$$
J(u, v)=\int_{0}^{T} g(t, y(t), u(t), v(t)) d t+\left\|y(T)-y^{*}\right\|^{2} \longrightarrow \min _{(u, v)}
$$

subject to

$$
\left\{\begin{array}{l}
\dot{y}=f(t, y(t), u(t), v(t)) \\
y\left(t_{0}\right)=y_{0}
\end{array}\right.
$$

where $y=(S, E, I, J, L, R)$ and $f$ is the right hand side of system (2). We analyze sufficient conditions for the existence of a solution to the optimal control problem (6). 
Theorem 3.1. There exists an optimal control pair $\left(u^{*}(t), v^{*}(t)\right)$, and corresponding solution $S^{*}, E^{*}, I^{*}, J^{*}, L^{*}, R^{*}$ to the state initial value problem (2) that minimizes $J(u, v)$ over $\Gamma$.

Proof: We refer to the conditions in Theorem III.4.1 and its corresponding corollary in [16]. The requirements there on the set of admissible controls and on the set of end conditions are clearly met here. The following nontrivial requirements from Fleming and Rishel's theorem are listed and verified below:

$A$ The set of all solutions to system (2) with corresponding control functions in $\Gamma$ (as given in equation (4)) is nonempty.

$B$ The state system can be written as a linear function of the control variables with coefficients dependent on time and the state variables.

$C$ The integrand $g$ in equation (6) is convex with respect to parameters $u$ and $v$ and additionally fulfills

$$
g(t, S, E, I, J, L, R, u, v) \geq c_{1}|(u, v)|^{\tau}-c_{2},
$$

where $c_{1}>0$ and $\tau>1$.

In order to establish condition A, we refer to Theorem 3.1 by Picard-Lindelöf $[8,10]$. If the solutions of the state equations are a priori bounded and if the state equations are continuous and Lipschitz-continuous in the state variables, then there is a unique solution corresponding to every admissible control pair in $\Gamma$.

It is easy to see that the state system is continuous and bounded. It is also straightforward to show the boundedness of the partial derivatives with respect to the state variables in the state system, which establishes that the system is Lipschitz-continuous with respect to the state variables (see [7], page 248). This completes the proof, thus condition $A$ holds.

Condition $B$ is verified by observing the linear dependence of the state equations on controls $u$ and $v$.

Finally, to verify condition $C$ we note that the integrand $g$ of the objective functional is clearly convex in the controls. To prove the bound on $g$ we note that by the definition of $u$ and $v$, we have $C_{1} u^{2} \leq C_{1} u \leq C_{1}$, and thus, $C_{1} u^{2}-C_{1} \leq$ $C_{1} u-C_{1} \leq 0$; therefore,

$$
\begin{aligned}
g(t, S, E, I, J, L, R, u, v) & =B_{1}\left(d_{1} I(t)+d_{2} J(t)+d_{3} L(t)\right)+B_{2} I(t)+C_{1} u(t)+D_{1} v(t) \\
& -C_{2} \log (1-u(t))-D_{2} \log (1-v(t)) \\
& \geq \min \left(C_{1}, D_{1}\right)(u+v)-C_{1} \\
& \geq \min \left(C_{1}, D_{1}\right)\left(u^{2}+v^{2}\right)-C_{1}
\end{aligned}
$$

This completes the proof. 


\subsection{Characterization of optimal controls}

Let us consider an optimal control problem having the form

$$
J(u, v)=\int_{0}^{T} g(t, y(t), u(t), v(t)) d t+\left\|y(T)-y^{*}\right\| \longrightarrow \min _{(u, v)}
$$

subject to

$$
\left\{\begin{array}{l}
\dot{y}=f(t, y(t), u(t), v(t)) \\
y\left(t_{0}\right)=y_{0}
\end{array}\right.
$$

and constraints

$$
\begin{aligned}
& u(t) \geq 0, \\
& v(t) \geq 0 .
\end{aligned}
$$

Pontryagin's Maximum principle [34] allows to utilize costate functions to transform the optimization problem to the problem of determining the pointwise minimum relative to $u^{*}$ and $v^{*}$ of the Hamiltonian. The Hamiltonian is built from the cost functional (4) and the controlling dynamics (2) derive the optimality conditions:

$$
\begin{aligned}
& H(t, S, E, I, J, L, R, u, v)=B_{1}\left(d_{1} I(t)+d_{2} L(t)+d_{3} J(t)\right)+B_{2} I(t)+C_{1} u(t) \\
& -C_{2} \log (1-u(t))+D_{1} v(t)-D_{2} \log (1-v(t))+\lambda_{1}(\Lambda-\nu(I, J, L) S-\mu S) \\
& +\lambda_{2}\left(\left(1-p_{1}-p_{2}\right) \nu(I, J, L) S+\rho J+\sigma_{2} \nu(I, J, L) R-\sigma_{1}\left(1-r_{1}-v(t)\right) \nu(I, J, L) E-A_{1} E\right) \\
& +\lambda_{3}\left(\left(p_{1}+p_{2} u(t)\right) \nu(I, J, L) S+(1+u(t)) \delta L+(1+u(t)) \theta J+\gamma R\right) \\
& +\lambda_{3}\left(h\left(1-r_{1}-v(t)\right)\left(k+\sigma_{1} \nu(I, J, L)\right) E-A_{2}(t) I\right) \\
& +\lambda_{4}\left(p_{2}(1-u(t)) \nu(I, J, L) S+(1-h)\left(1-r_{1}-v(t)\right)\left(k+\sigma_{1} \nu(I, J, L)\right) E-A_{3} J,\right) \\
& +\lambda_{5}\left(\alpha(1-u(t)) I-A_{4} L\right)+\lambda_{6}\left(r_{2} I+\omega L-\sigma_{2} \nu(I, J, L) R-A_{5} R\right)
\end{aligned}
$$

where the $\lambda_{i}, i=1, \cdots, 6$ are the associated adjoints for the states $S, E, I, J, L$ and $R$. The optimality system of equations is found by taking the appropriate partial derivatives of the Hamiltonian (8) with respect to the associated state variable.

The following theorem is a consequence of the maximum principle.

Theorem 3.2. Given an optimal control pair $\left(u^{*}, v^{*}\right)$ and corresponding solutions to the state system $S^{*}, E^{*}, I^{*}, J^{*}, L^{*}, R^{*}$, that minimize the objective functional (4), there exist adjoint variables $\lambda_{1}, \lambda_{2}, \lambda_{3}, \lambda_{4}, \lambda_{5}$, and $\lambda_{6}$, satisfying

$$
\begin{aligned}
\frac{d \lambda_{1}}{d t} & =\mu \lambda_{1}-\left(\left(\beta_{1} I+\beta_{2} L\right) \frac{N-S}{N^{2}}+\beta_{3} J\right) \\
& \times\left(-\lambda_{1}+\left(1-p_{1}-p_{2}\right) \lambda_{2}+\left(p_{1}+p_{2} u(t)\right) \lambda_{3}+p_{2}(1-u(t)) \lambda_{4}\right) \\
& +\left(\beta_{1} I+\beta_{2} L\right) \frac{1}{N^{2}}\left[\sigma_{1}\left(1-r_{1}-v(t)\right) E\left(-\lambda_{2}+h \lambda_{3}+(1-h) \lambda_{4}\right)+\sigma_{2} R\left(\lambda_{2}-\lambda_{6}\right)\right]
\end{aligned}
$$




$$
\begin{aligned}
& \frac{d \lambda_{2}}{d t}=\left(\beta_{1} I+\beta_{2} L\right) \frac{S}{N^{2}}\left(-\lambda_{1}+\left(1-p_{1}-p_{2}\right) \lambda_{2}+\left(p_{1}+p_{2} u(t)\right) \lambda_{3}+p_{2}(1-u(t)) \lambda_{4}\right) \\
& +\left(\beta_{1} I+\beta_{2} L\right) \frac{R}{N^{2}} \sigma_{2}\left(\lambda_{2}-\lambda_{6}\right)+\mu \lambda_{2} \\
& -\left(1-r_{1}-v(t)\right)\left[\left(\left(\beta_{1} I+\beta_{2} L\right) \frac{N-E}{N^{2}}+\beta_{3} J\right) \sigma_{1}+k\right]\left(-\lambda_{2}+h \lambda_{3}+(1-h) \lambda_{4}\right) \\
& \frac{d \lambda_{3}}{d t}=-\left(S \frac{\beta_{1} N-\left(\beta_{1} I+\beta_{2} L\right)}{N^{2}}\right)\left(-\lambda_{1}+\left(1-p_{1}-p_{2}\right) \lambda_{2}+\left(p_{1}+p_{2} u(t)\right) \lambda_{3}+p_{2}(1-u(t)) \lambda_{4}\right) \\
& -\left(\frac{\beta_{1} N-\left(\beta_{1} I+\beta_{2} L\right)}{N^{2}}\right)\left[\sigma_{1}\left(1-r_{1}-v(t)\right) E\left(-\lambda_{2}+h \lambda_{3}+(1-h) \lambda_{4}\right)+\sigma_{2} R\left(\lambda_{2}-\lambda_{6}\right)\right] \\
& +\left(\mu+d_{1}\right) \lambda_{3}-r_{2}\left(\lambda_{6}-\lambda_{3}\right)-\alpha(1-u)\left(\lambda_{5}-\lambda_{3}\right)-B_{1} d_{1}-B_{2} \\
& \frac{d \lambda_{4}}{d t}=-B_{1} d_{2}-R\left(-\frac{\beta_{1} I+\beta_{2} L}{N^{2}}+\beta_{3}\right) \sigma_{2}\left(\lambda_{2}-\lambda_{6}\right) \\
& -S\left(-\frac{\left(\beta_{1} I+\beta_{2} L\right)}{N^{2}}+\beta_{3}\right)\left(-\lambda_{1}+\left(1-p_{1}-p_{2}\right) \lambda_{2}+\left(p_{1}+p_{2} u(t)\right) \lambda_{3}+p_{2}(1-u(t)) \lambda_{4}\right) \\
& -\left(\frac{-\left(\beta_{1} I+\beta_{2} L\right)}{N^{2}}+\beta_{3}\right) E \sigma_{1}\left(1-r_{1}-v(t)\right)\left(-\lambda_{2}+h \lambda_{3}+(1-h) \lambda_{4}\right) \\
& -\rho\left(\lambda_{2}-\lambda_{4}\right)+\left(\mu+d_{2}\right) \lambda_{4}-\theta(1+u(t))\left(\lambda_{3}-\lambda_{4}\right) \\
& \frac{d \lambda_{5}}{d t}=-\left(S \frac{\beta_{2} N-\left(\beta_{1} I+\beta_{2} L\right)}{N^{2}}\right)\left(-\lambda_{1}+\left(1-p_{1}-p_{2}\right) \lambda_{2}+\left(p_{1}+p_{2} u(t)\right) \lambda_{3}+p_{2}(1-u(t)) \lambda_{4}\right) \\
& -\left(\frac{\beta_{2} N-\left(\beta_{1} I+\beta_{2} L\right)}{N^{2}}\right)\left[\sigma_{1}\left(1-r_{1}-v(t)\right) E\left(-\lambda_{2}+h \lambda_{3}+(1-h) \lambda_{4}\right)+\sigma_{2} R\left(\lambda_{2}-\lambda_{6}\right)\right] \\
& +\left(\mu+d_{3}\right) \lambda_{5}-\delta(1+u(t))\left(\lambda_{3}-\lambda_{5}\right)-\omega\left(\lambda_{6}-\lambda_{5}\right)-B_{1} d_{3} \\
& \frac{d \lambda_{6}}{d t}=\frac{S\left(\beta_{1} I+\beta_{2} L\right)}{N^{2}}\left(-\lambda_{1}+\left(1-p_{1}-p_{2}\right) \lambda_{2}+\left(p_{1}+p_{2} u(t)\right) \lambda_{3}+p_{2}(1-u(t)) \lambda_{4}\right) \\
& -\left(\left(\beta_{1} I+\beta_{2} L\right) \frac{N-R}{N^{2}}+\beta_{3} J\right) \sigma_{2}\left(\lambda_{2}-\lambda_{6}\right)+\mu \lambda_{6}-\gamma\left(\lambda_{3}-\lambda_{6}\right) \\
& +\frac{E\left(\beta_{1} I+\beta_{2} L\right)}{N^{2}} \sigma_{1}\left(1-r_{1}-v(t)\right)\left(-\lambda_{2}+h \lambda_{3}+(1-h) \lambda_{4}\right)
\end{aligned}
$$

with terminal conditions

$$
\begin{aligned}
& \lambda_{1}(T)=0, \quad \lambda_{2}(T)=0, \quad \lambda_{3}(T)=2 D_{3}\left(I(T)-I_{0}\right), \\
& \lambda_{4}(T)=2 D_{3}\left(J(T)-J_{0}\right), \quad \lambda_{5}(T)=2 D_{3}\left(L(T)-L_{0}\right), \quad \lambda_{6}(T)=0 .
\end{aligned}
$$

Furthermore, we may characterize the optimal pair by the piecewise continuous 
functions

$$
\begin{aligned}
& u^{*}=\max \left(0,1+\frac{C_{2}}{C_{1}+\left(\nu(I, J, L) S p_{2}+\theta J\right)\left(\lambda_{3}-\lambda_{4}\right)+(\delta L+\alpha I)\left(\lambda_{3}-\lambda_{5}\right)}\right), \\
& v^{*}=\max \left(0,1+\frac{D_{2}}{D_{1}-\left(\sigma_{1} \nu(I, J, L)+k\right) E\left(-\lambda_{2}+h \lambda_{3}+(1-h) \lambda_{4}\right)}\right) .
\end{aligned}
$$

Proof: The result follows from a direct application of a version of Pontryagin's Maximum Principle for bounded controls [25]. The differential equations governing the adjoint variables are obtained by differentiation of the Hamiltonian function (8), evaluated at the optimal control. Then the adjoint system can be written as dictated by the Maximum Principle, by the equations

$$
\begin{aligned}
\frac{d \lambda_{1}}{d t} & =-\frac{\partial H}{\partial S}, \quad \lambda_{1}(T)=0, \quad \frac{d \lambda_{2}}{d t}=-\frac{\partial H}{\partial E}, \quad \lambda_{2}(T)=0, \\
\frac{d \lambda_{3}}{d t} & =-\frac{\partial H}{\partial I}, \quad \lambda_{3}(T)=2 D_{3}\left(I(T)-I_{0}\right), \\
\frac{d \lambda_{4}}{d t} & =-\frac{\partial H}{\partial J}, \quad \lambda_{4}(T)=2 D_{3}\left(J(T)-J_{0}\right), \\
\frac{d \lambda_{5}}{d t} & =-\frac{\partial H}{\partial L}, \quad \lambda_{5}(T)=2 D_{3}\left(L(T)-L_{0}\right), \\
\frac{d \lambda_{6}}{d t} & =-\frac{\partial H}{\partial R}, \quad \lambda_{6}(T)=0,
\end{aligned}
$$

evaluated at the optimal control pair $\left(u^{*}, v^{*}\right)$ and corresponding states. This results in the stated adjoint system (9)-(14).

Finally, the optimality conditions require that,

$$
\frac{\partial H}{\partial u}=\frac{\partial H}{\partial v}=0,
$$

for the optimal pair $\left(u^{*}, v^{*}\right)$, on the interior of the control set, and this condition is simplified in equations (16) with special attention on control arguments involving the bounds on the controls as defined with $\Gamma$ in equation (4).

\section{Numerical simulation of optimal controls}

Optimal educational and chemoprophylaxis strategies are obtained by solving the optimality system, consisting of 12 ODEs from the state and adjoint equations above. An iterative method is used for solving the optimality system. The state system with an initial guess is solved forward in time and then the adjoint system is solved backward using the transversality conditions. Note that, since the model is not stiff, a number of numerical scheme for ODEs in the literature may solve forward as well as backward the optimality problem which is used here. From model (2), we generated the state values of different epidemiological classes for the year 2015 using data in Tables 1 and .2. The initial values are thus defined as

$$
\begin{aligned}
& S_{0}=4,950,585, \quad E_{0}=15,594,821, \quad I_{0}=21,392, \quad J_{0}=13,009, \\
& L_{0}=3,723 \quad R_{0}=139,455 \text { and } N_{0}=20,722,985,
\end{aligned}
$$


representing model predictions for years 2015. These initial population sizes will be used throughout the rest of following paragraph.

For the sake of realism, we have chosen to implement the optimal control over 10 years on model (2), because we assume that it remains realistic enough to reproduce the dynamics of TB in Cameroon over this period, also according to new development strategy presented by the Cameroonian government. Another approach for optimal control could be the infinite time arising case, to follow the longterm optimal control of TB in Cameroon. However, since parameters can change, it is suitable on stochastic systems. We have chosen a 10 years horizon optimal control approach to describe the impact of educational campaign on TB dynamics. However, most numerical simulation will be performed on 25 years, to minimize the influence of the final time arising over the 10 first years.

Throughout the iterations, a lower bound $u_{0}=v_{0}=0$ will be keep for the control through a projection of $u$ and $v$ on the interval $\left[0, u_{m}\right] \times\left[0, v_{m}\right]$ where $u_{m}=v_{m}=1$.

Step size. An appropriate ODE integrator as well as an efficient optimal control update should exert some adaptive control over its own progress, making appropriate changes in its step-size. Usually the purpose of this adaptive stepsize control is to achieve some predetermined accuracy in the solution with minimum computational effort. Implementation of adaptive step-size control requires that the stepping algorithm return information about its performance, most important, an estimate of its truncation error. Obviously, the calculation of this information will add to the computational overhead, but the investment will generally be repaid. In this work, an adaptive step-size control implemented in the software Dopri5 [11] will be used to solve forward the states equations and backward the adjoint equations. The control at the end of each iteration have been chosen to be updated through $u^{k}=u^{*}$ when $u^{*}$ is the value from the characterizations in equation (16). The same type of update is used for the second control function $v$ update $\left(v^{k}=v^{*}\right)$. In contrast with the traditional convex combination used frequently in the literature to implement the FBSM $[26,25]$, this update appeared to be faster and lead to the same result. The choice of the control updates is justify by the speed of convergence to the optimal solution which happened fast (after less than 3 minutes of computation). Time points chosen by adaptive step-size control generally do not agree with discretization points of the controls. An integrator with dense output is used to solve the problem. It constructs an interpolation polynomial based on the available solution such that accuracy is not destroyed.

Stopping criteria. The optimal control iterations continue until convergence when the relative update between all state variables, the adjoint functions and the control functions are less than a defined value $T O L$, i.e.

$$
\min _{i} \frac{\left\|x_{i}^{k}-x_{i}^{k-1}\right\|}{\left\|x_{i}^{k}\right\|}<T O L
$$


where $x_{i}$ is either a state variable, the adjoint function or the control. The value $T O L=0.0001$ is used in this numerical simulation. Parameter values are defined in Table 1 and data in Table .2 will be divided by 10000 in order to obtain a well scaled system.

\subsection{Optimal educational strategy}

Let us first focus on the optimal educational strategy for the population of Cameroon. Numerical simulations are performed using the FBSM for $D_{1}=$ $D_{2}=0$ when $v=0$. Then, the optimal control problem is defined by

$$
\begin{aligned}
J(u, v)= & \int_{0}^{T} g(t, y(t), u(t)) d t+\left\|y(T)-y^{*}\right\|^{2} \longrightarrow \min _{u} \\
& \text { subject to } \\
& \left\{\begin{array}{l}
\dot{y}=f(t, y(t), u(t)), \\
y\left(t_{0}\right)=y_{0} .
\end{array}\right.
\end{aligned}
$$

where $y=(S, E, I, J, L, R)$,

$$
g(t, y, u)=B_{1}\left(d_{1} I(t)+d_{2} J(t)+d_{3} L(t)\right)+B_{2} I(t)+C_{1} u(t)-C_{2} \log (1-u(t))
$$

and $f$ the right hand side of system (2).

Figure 3 shows a time optimal educational schedule for $T=10$ years. Here and on all following figures, S, E, I, J, L and R stand for susceptible, latently infected, diagnosed infectious, undiagnosed infectious, lost sight and recovered population when $u$ is the effort in educating and diagnosing the population. It appears that the disease burden will indeed decrease due to the influence of education $u$. Figure 3 (c) reveals an increase of the number of diagnosed infectious for one year as result of the campaign. After one year, the number of undiagnosed infectious drops and be less than the expected number after 3 years without the control. As a result of the decrease of the number of infectious, the susceptible population will increase and the recovered population will decrease (Figure 3 (a) and (f)). The control function in Figure $3(\mathrm{u})$ is continuous and decreasing with respect to time.

Figure 4 illustrates the convergence of the FBSM throughout the iterations when only education is applied in the population. It appears from the Figure that at the end of the iterations, the norm of the Hamiltonian's gradient goes very close to 0 .

\subsection{Optimal chemoprophylaxis strategy}

Setting $C_{1}=C_{2}=0$ and $u=0$ in system (2), the corresponding cost function is defined by

$$
\begin{aligned}
J_{v}(v) & =\int_{0}^{T}\left\{B_{1}\left(d_{1} I(t)+d_{2} J(t)+d_{3} L(t)\right)+B_{2} I(t)+D_{1} v(t)-D_{2} \log (1-v(t))\right\} d t \\
& +D_{3}\left\|y(T)-y^{*}\right\|^{2}
\end{aligned}
$$

Figure 5 presents the numerical results of a chemoprophylaxis optimal control on the dynamics of $\mathrm{TB}$ in $T=10$ years. It reveals that the population of 


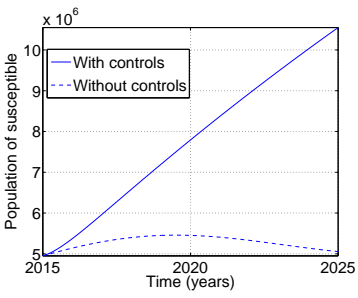

(a) $\mathrm{S}$

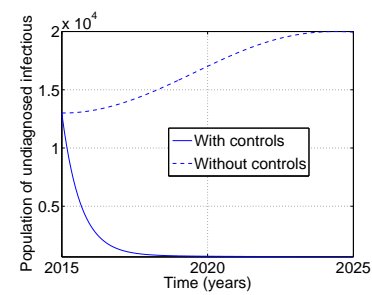

(d) $\mathrm{J}$

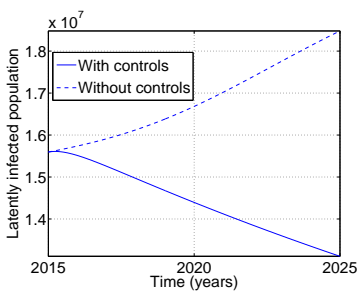

(b) $\mathrm{E}$

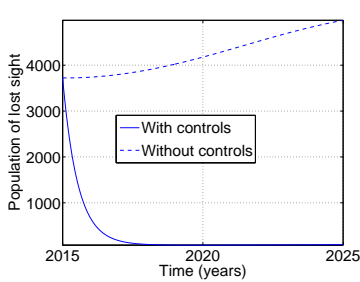

(e) L

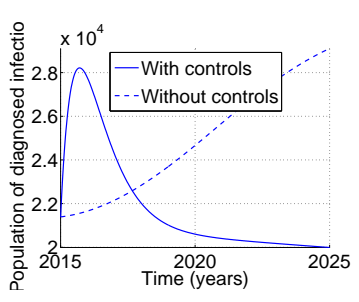

(c) I

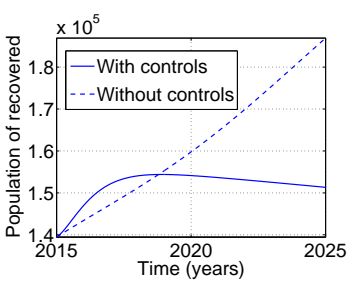

(f) $\mathrm{R}$

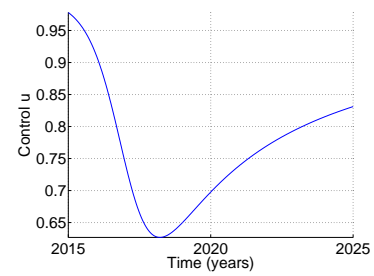

(g) $\mathrm{u}$

Figure 3: Simulations of the TB model (2) showing the effect of optimal educational and diagnosis campaign rates on the TB infection.

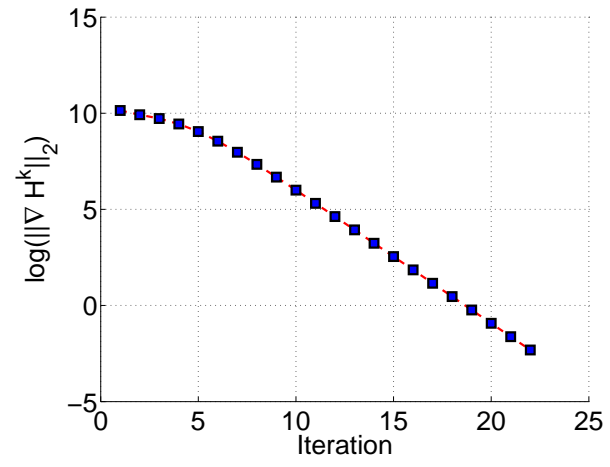

Figure 4: Evolution of the norm of the Hamiltonian's gradient throughout iterations corresponding to the educational only optimal strategy.

infectious will drop for five years when the control strategy will be implemented, but increase again after. The reverse effect will be observed for the susceptible population. Results in Figure 5 show that chemoprophylaxis of latently infected 


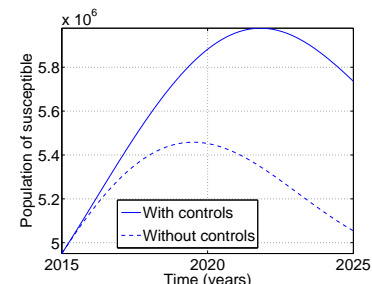

(a) $\mathrm{S}$

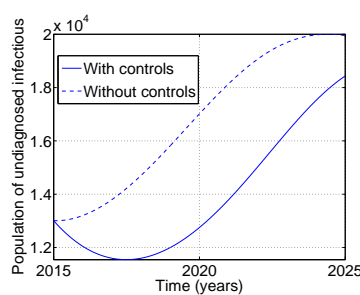

(d) $\mathrm{J}$

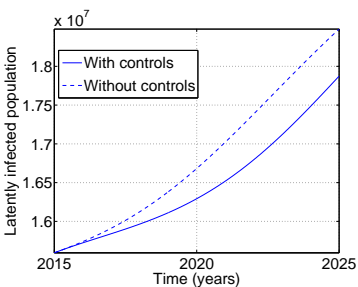

(b) $\mathrm{E}$

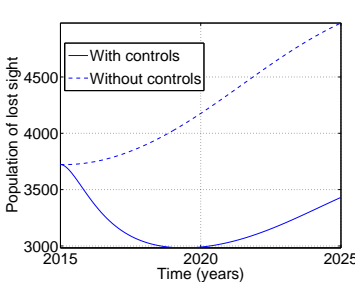

(e) $\mathrm{L}$

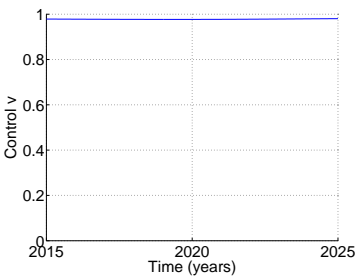

(g) $\mathrm{v}$

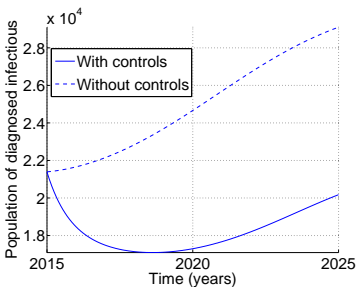

(c) I

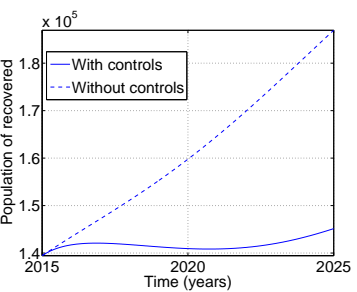

(f) $\mathrm{R}$

Figure 5: Simulations of the TB model (2) showing the effect of optimal chemoprophylaxis and treatment rates against constant education and treatment rates on the infected population.

population may delay the TB propagation, but cannot eradicate the disease. The chemoprophylaxis strategy will decrease the number of infected population by more than 40 percent during the 10 years of optimal control. The fact is that chemoprophylaxis does not reduce the contact between infectious and susceptible population, but only minimizes the evolution to the disease for the latently infected population. In the presence of fast progression, this strategy can not lead to a satisfactory way for reducing TB if applied without further efforts.

Similarly to Figure 4, an analysis of the Hamiltonian can reveal the convergence of the FBSM.

\subsection{Optimal education and chemoprophylaxis}

Let us find now the optimal schedule for both education and chemoprophylaxis on the population using data from Cameroon. For the Figures presented here, we assume that the weight factor $C_{1}$ associated with control $u$ is smaller than $D_{1}$ which is associated with control $v$. This assumption is based on fol- 
lowing facts: the cost associated with chemoprophylaxis $v$ will include the cost of screening and treatment programs, and the cost associated with $u$ including those of educating people about the TB diagnosis in the hospital or sending people to watch the patients to finish their treatment. Treating an infectious TB individual takes longer (by several months) than treating a latent TB individual [21].

To minimize the total number of TB induced death, the optimal control $u$ decreases during the $T=25$ years and $v$ is also decreasing from the upper bound, while the steadily decreasing value for $u$ is applied over the most of the simulated time, $T=25$ years. The initial conditions used in this case are extracted from numerical simulations of the model corresponding to year 2015 . Combining chemoprophylaxis and education, the burden of TB can be reduced by $80 \%$ in 10 years.

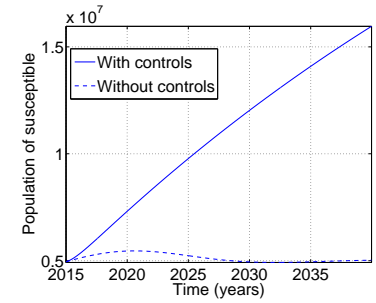

(a) $\mathrm{S}$

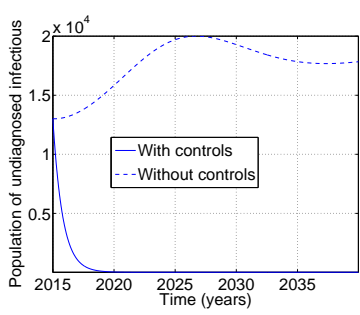

(d) $\mathrm{J}$

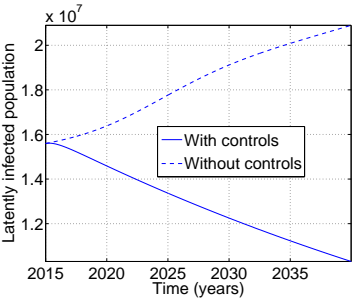

(b) $\mathrm{E}$

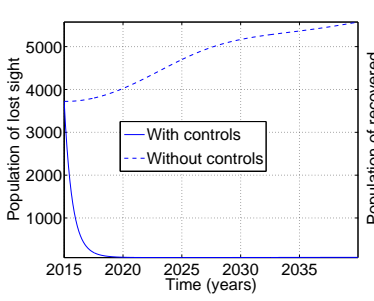

(e) $\mathrm{L}$

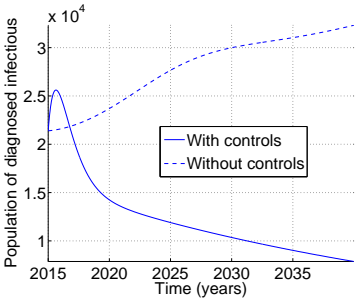

(c) I

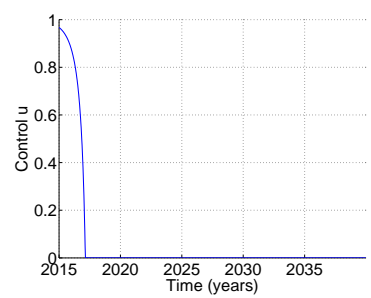

(g) u

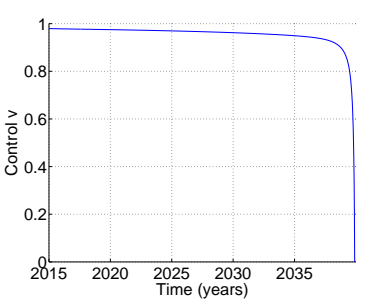

(h) v

Figure 6: Time series of model (2) results from optimal education and diagnosis campaign and chemoprophylaxis strategies (dashed lines) compared with of no education and chemoprophylaxis control strategies (plained lines). Without education and chemoprophylaxis, the number of infectious increases little and the number of susceptible decreases little. In the presence of education and chemoprophylaxis, the opposite effect is observed. 
The convergence of the FBSM throughout the iterations when both control strategies are applied has been again verified through the Hamiltonian gradient's norm.

\subsection{Impact of the objective functional parameters}

The sensitivity of the optimal control with respect to constants such as $C_{1}$, $C_{2}, D_{1}$ and $D_{2}$ is a well known and recurrent question. In this paragraph, we present the optimal control solutions for several values of $C_{1}, C_{2}, D_{1}$, and $D_{2}$. Figure 7 illustrates how the optimal control strategies depend on the parameter $C_{1}, C_{2}, D_{1}$, and $D_{2}$, which are the coefficients of the logarithmic and the linear part of the objective functional defined in equation (4). Coefficients $D_{1}$, and $D_{2}$ show the influence of the logarithmic non-linear term on other control parameters. These parameters values may vary from place to place depending on many factors including living conditions and culture. In Figure 7 and 8 the controls $u$ and $v$ are plotted as a function of time for the 6 different values of $C_{1}=C_{2} \in\{1,21,41,61,81,101\}$ and $D_{1}=D_{2} \in\{1,21,41,61,81,101\}$. Other parameters are fixed as in Tables 1 and .2. Figure 7 and 8 show that the coefficients play a decreasing role on the control while state values remain almost the same as $C_{1}=C_{2}$ and $D_{1}=D_{2}$ increases. The values $C_{1}=C_{2}$ and $D_{1}=D_{2}$ have been chosen to express the particular case where the amount of money for educational and chemoprophylaxis campaigns are available in different proportion.

Figure 11 presents the dynamics of controls as function of non linearities on the cost function. To analyse the impact of the logarithmic part on the cost function, we assumed $C_{2}=D_{2} \longrightarrow 0$, which are the coefficients of the logarithmic part of the objective functional defined in equation (4). In the case $C_{1}=D_{1}=20$ have been used and for different values of $C_{2}=D_{2} \in$ $(1 e-9,0.01)$. The result presented in figure $11 u_{1}$ and $v_{1}$ illustrates that the non logarithmic expression prevent the control for reaching the maximum value 1. When $C_{2}=D_{2} \longrightarrow 0$, the obtained results tends to the bang-bang control. When $C_{1}=D_{1} \longrightarrow 0$ (see, figure $u_{2}$ and $v_{2}$ ), the control does not reach the maximum value.

Figure 10 illustrates the convergence of the FBSM throughout the iterations. It appears that the linear convergence of the FBSM is barely affected by parameters changes.

\subsection{Impact of the final time}

The sensitivity of the optimal control solution with respect to the final time $T$ is also a well known and recurrent question. In this paragraph, we present the optimal control solutions for several values of $T$. Figure 11 illustrates how the optimal control strategies depend on the final time $T$ of the control strategies. It comes out that the educational campaign is sensitive on with respect to the final time arising optimal control, however, an analysis of the ten year optimal control strategy is enough to ensure a low number of undiagnosed in the population. It can be observed that for $T=15$, a new campaign have to be implemented at the 


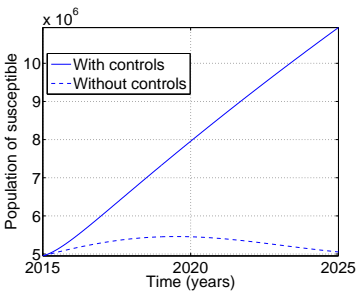

(a) $\mathrm{S}$

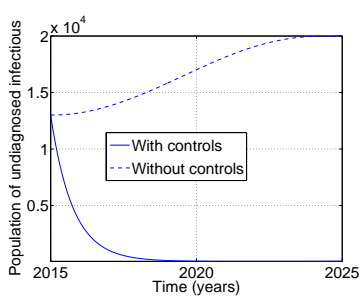

(d) $\mathrm{J}$

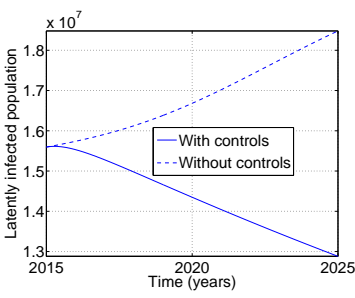

(b) $\mathrm{E}$

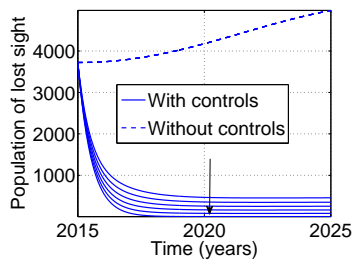

(e) $\mathrm{L}$

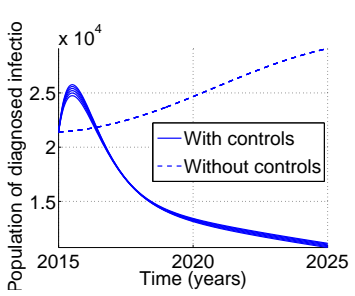

(c) I

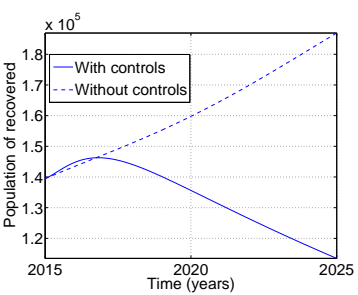

(f) $\mathrm{R}$

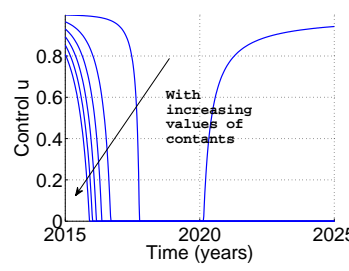

(g) u

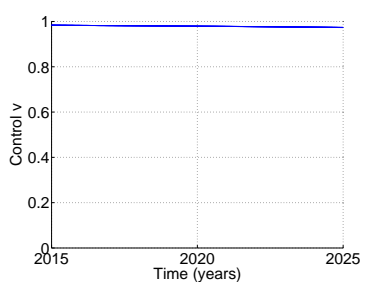

(h) v

Figure 7: Evolution of model (2) from optimal education and chemoprophylaxis strategies, dashed lines, compared with that of no education and chemoprophylaxis control strategies (plained lines) for $C_{1}=C_{2} \in\{1,21,41,61,81,101\}$. Other parameters are presented in Tables 1 and .2. A large change on $u$ and $v$ with the constance values is observed.

9th years. The same pulse have been observed at the end of each control. This can be explained by the end condition which ensure the number of undiagnosed to be as closed as possible to zero. It can be observed that the chemoprophylaxis strategy decreases to zero depending on the final time of the control. However, the optimal solution of system (2) is not sensitive with respect to the final time. This ensures the optimal solution to be reached regardless of the final time used for the implementation.

\section{Conclusion}

The TB incidence rates have been declining since 2010 in Cameroon, namely due to prevention and treatment policies that have been applied in the last years through "DOTS" and "Stop TB" programs. The reduction of mortality and incidence rates depend on the effort at country level to implement control 


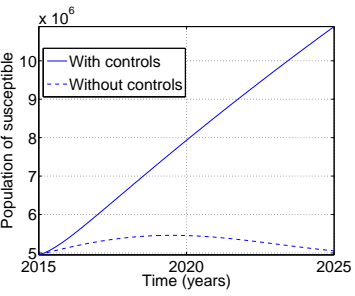

(a) $\mathrm{S}$

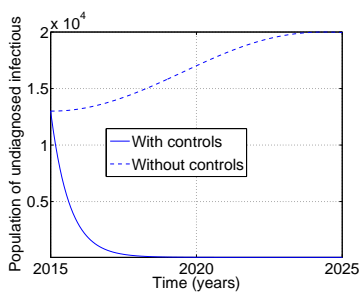

(d) $\mathrm{J}$

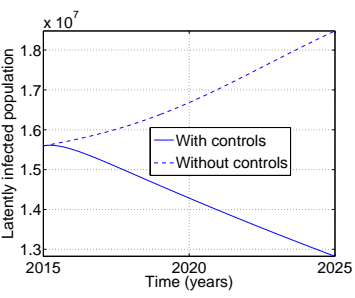

(b) $\mathrm{E}$

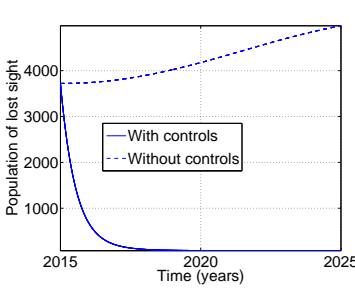

(e) L

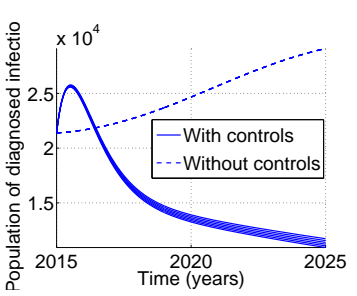

(c) I

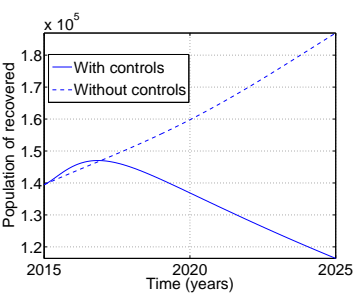

(f) $\mathrm{R}$

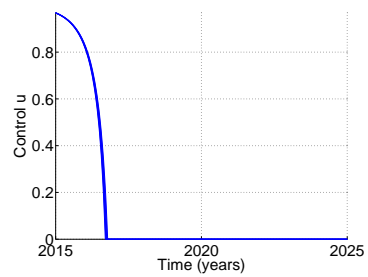

(g) $\mathrm{u}$

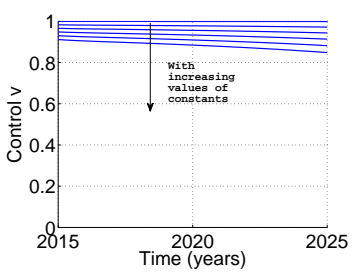

(h) $\mathrm{v}$

Figure 8: Evolution of model (2) result from optimal education and chemoprophylaxis strategies, dashed lines, compared with that of no education and chemoprophylaxis control strategies (plained lines) for $D_{1}=D_{2} \in\{1,21,41,61,81,101\}$. Other parameters are presented in Tables 1 and .2. One observes a large change on $u$ and $v$ with the constance values.

policies. One of the most successful TB control methods implemented in the world is the DOTS strategy. However, in many sub-Saharan countries, the number of estimated cases of TB infected persons is still very high compared to the number of diagnosed cases. This made the DOTS program and the new "Stop TB" program not fully implemented.

In this paper we presented a mathematical model for $\mathrm{TB}$, which takes into account population of undiagnosed and lost sight infectious, with the aim of controlling TB propagation through these classes. Optimal time-dependent prevention policies which consider also the execution cost, are proposed. We implemented different numerical approaches and we observed that the pattern of TB can be controlled if in addition of the current "Stop TB" strategy, an educational and diagnosis campaign, and chemoprophylaxis of latently infected individuals was performed on the population. The model take into account the actual data on TB in Cameroon and the rate of success of the treatment. Our 


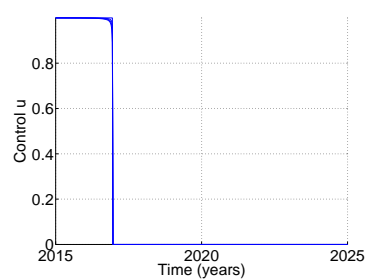

(a) $u_{1}$

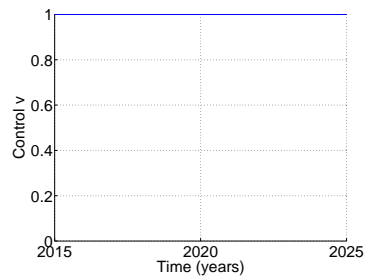

(c) $v_{1}$

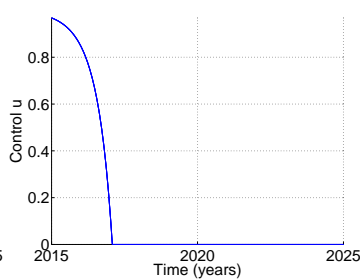

(b) $u_{2}$

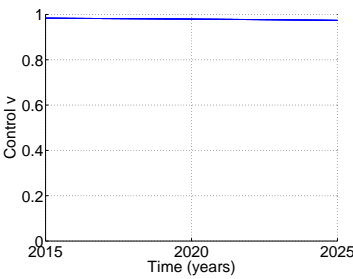

(d) $v_{2}$

Figure 9: Evolution of optimal education and chemoprophylaxis strategies for $C_{2}=D_{2} \in$ $(1 e-9,0.1)$, compared with the case $C_{1}=D_{1} \in(1 e-9,0.1)$. Other parameters are presented in Tables 1 and .2.
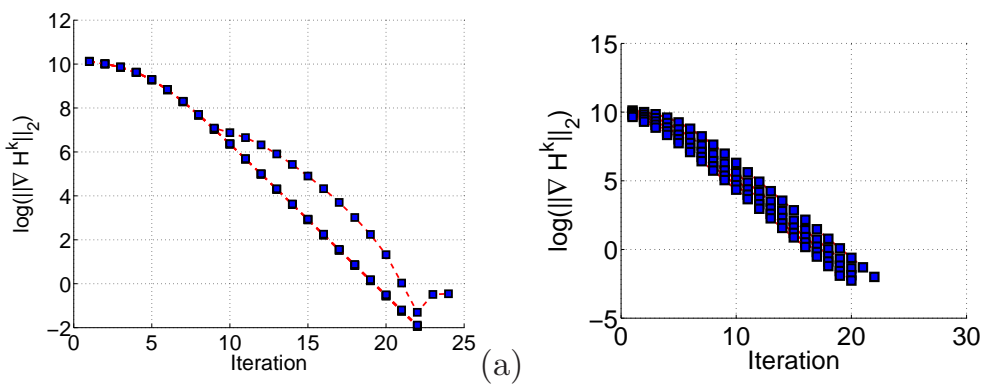

(b)

Figure 10: Evolution of the norm of the Hamiltonian's gradient with respect to iterations.

results show that the implementation of the combined effect of education and diagnose campaign with chemoprophylaxis of latently infected may reduce by $80 \%$ the TB burden in 10 years. The FBSM converges linearly and robustly. Numerical simulation showed that change on model parameters does not influence the convergence of the FBSM. However, a change on the cost function parameters and the discretization affects the control solution. A possible future issue consists of quantifying the impact of cost-functional parameters on the control function and to compare different direct and indirect methods for optimal control. The same method can be applied to TB dynamics with seasonality or considering a co-infection model of TB with HIV/AIDS and diabetes. From these analysis, it may be possible to analyze combining strategies to different kind of diseases. 


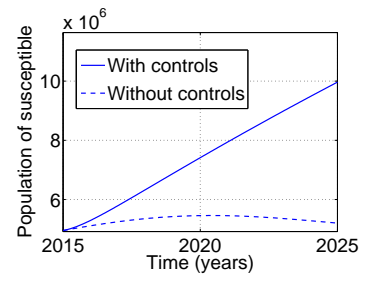

(a) $\mathrm{S}$

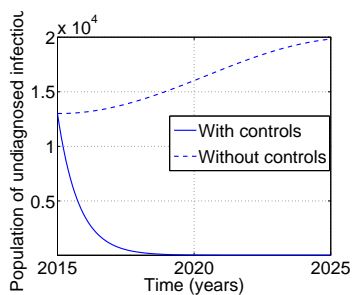

(d) $\mathrm{J}$

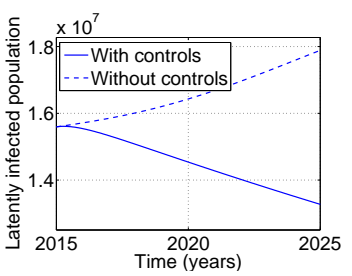

(b) $\mathrm{E}$

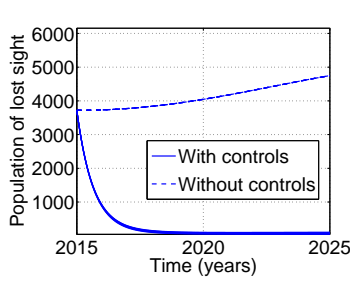

(e) L

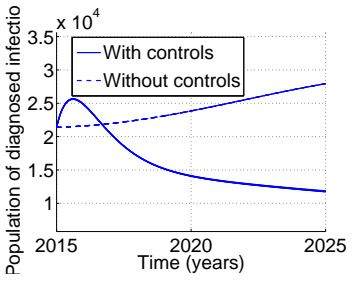

(c) I

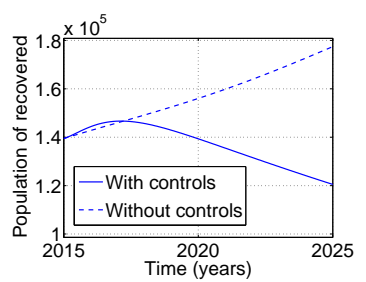

(f) $\mathrm{R}$

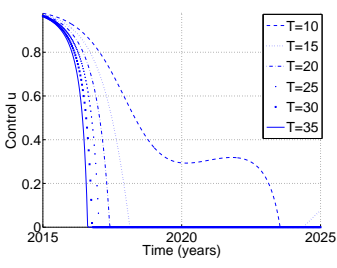

(g) $\mathrm{u}$

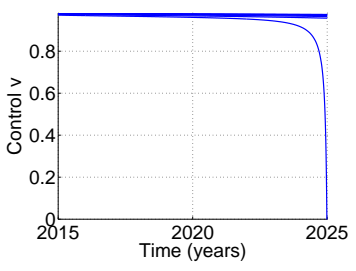

(h) $\mathrm{v}$

Figure 11: Evolution of model (2) result from optimal education and chemoprophylaxis strategies, compared with the case of no education and chemoprophylaxis control strategies for $T \in\{10,15,20,25,30,35\}$. Other parameters are presented in Tables 1 and .2 .

\section{Acknowledgment}

We thank the referees for the careful reading of this manuscript, the questions they posed and suggestions they offered. As a result, this paper is significantly improved.

\section{References}

[1] F. B. Agusto. Optimal chemoprophylaxis and treatment control strategies of a tuberculosis transmission model. World Journal of Modelling and Simulation, 5(3):163-173, 2009.

[2] N. Bacaër, R. Ouifki, C. Pretorius, R. Wood, and B. Williams. Modeling the joint epidemics of TB and HIV in a South African township. J Math Biol, 57(4):557-93, Oct. 2008. 


\begin{tabular}{lll}
\hline \hline Parameters & Value & Source \\
\hline \hline$B_{1}$ & 3000.00 USD per human death & {$[30]$} \\
$B_{2}$ & 563.00 USD per person treatment & {$[17]$} \\
$C_{1}$ & 200000 USD per proportion of effort & Assumed \\
& to educated people & \\
$C_{2}$ & 200000 USD per logarithmic of the proportion of & Assumed \\
& people to educate & \\
$D_{1}$ & 250000 USD per proportion of people & Assumed \\
& treated by chemoprophylaxis & \\
$D_{2}$ & 250000 USD per logarithmic of level of & Assumed \\
& chemoprophylaxis to implement & \\
$D_{3}$ & 5 per unity of distance between $y(T)$ and $E_{0}$ & Assumed \\
\hline \hline
\end{tabular}

Table .2: Numerical values for the cost functional parameters.

[3] Y. Balabanova, V. Tchernyshev, I. Tsigankov, S. Maximova, N. Mikheeva, L. Fedyukovitch, S. Kuznetsov, I. Fedorin, and F. Drobniewski. Analysis of undiagnosed tuberculosis-related deaths identified at post-mortem among hiv-infected patients in russia: a descriptive study. BMC Infectious Diseases, 11(1):276, 2011.

[4] M. Begon, M. Bennett, R. G. Bowers, N. P. French, S. M. Hazel, and J. Turner. A clarification of transmission terms in host-microparasite models : numbers, densities and areas. Epidemiol. Infect., 129:147-153, 2002.

[5] S. M. Blower, P. Small, and P. Hopewell. Control strategies for tuberculosis epidemics: new method for old problem. Science, 273:497-500, 1996.

[6] L. Bolzoni, V. Tessoni, M. Groppi, and G. A. Leo. React or wait: which optimal culling strategy to control infectious diseases in wildlife. Journal of Mathematical Biology, pages 1-25, 2013.

[7] E. A. Coddington. An Introduction to Ordinary Differential Equations. Prentice-Hall Inc, 1961.

[8] E. A. Coddington and N. Levinson. Theory of Ordinary Differential Equations. Mc-Graw Hill Co. Inc.,, 1955.

[9] L. de Pillis, W. Gu, K. Fister, T. Head, K. Maples, A. Murugan, T. Neal, and K. Yoshida. Chemotherapy for tumors: An analysis of the dynamics and a study of quadratic and linear optimal controls. Mathematical Biosciences, 209(1):292 - 315, 2007.

[10] P. Deuflhard and F. Bornemann. Scientific computing with ordinary differential equations., volume 42. Texts in Applied Mathematics, Springer, Berlin, 2002. 
[11] J. R. Dormand and P. J. Prince. A family of embedded Runge-Kutta formulae. J of Comput and Appl Math, 6:19-26, 1980.

[12] DTBE. Fact sheets. Technical report, Division of Tuberculosis Elimination, Centers for Disease Control and Prevention, 2011.

[13] C. Dye, K. Lönnroth, E. Jaramillo, B. G. Williams, and M. Raviglione. Trends in tuberculosis incidence and their determinants in 134 countries. Bull. World Health Organ., 87(9):683-91, Sept. 2009.

[14] Y. Emvudu, R. Demasse, and D. Djeudeu. Optimal control of the lost to follow up in a tuberculosis model. Comput. Math. Methods Med., 00:1-12, 2011.

[15] K. R. Fister, S. Lenhart, and J. McNally. Optimizing Chemotherapy in an HIV. Electronic Journal of Differential Equations, 32(32):1-12, December 1998.

[16] W. H. Fleming and R. W. Rishel. Deterministic and Stochastic Optimal Control and Applications of Mathematics. 1. Springer-Verlag, 1975.

[17] M. N. Gourevitch, P. Alcabes, W. C. Wasserman, and P. S. Arno. Costeffectiveness of directly observed chemoprophylaxis of tuberculosis among drug users at high risk for tuberculosis. Int J Tuberc Lung Dis., 2(7):53140, July 1998.

[18] E. Hansen and T. Day. Optimal control of epidemics with limited resources. Journal of Mathematical Biology, 62(3):423-451, 2011.

[19] K. Hattaf, M. Rachik, S. Saadi, Y. Tabit, and N. Yousfi. Optimal control of tuberculosis with exogenous reinfection. Applied Mathematical Sciences, $3(5): 231-240,2009$.

[20] H. R. Joshi. Optimal control of an HIV immunology model. Optimal Control Applications \& Methods, 23:199-213, 2002.

[21] E. Jung, S. Lenhart, , and Z. Feng. Optimal control of treatments in a twostrain tuberculosis. Discrete and Continuous Dynamical Systems. Series B, 164:183-201, 2002.

[22] D. Kirschner, S. Lenhart, and S. Serbin. Optimal control of the chemotherapy of hiv. Math. Biol., 35:775-792, 1997.

[23] V. Kumar, A. K. Abbas, N. Fausto, and R. N. Mitchell. Basic Pathology (8th ed.), volume ISBN 978-1-4160-2973-1. Saunders Elsevier, 2007.

[24] R. Laxminarayan, E. Klein, C. Dye, K. Floyd, S. Darley, and O. Adeyi. Economic benefit of tuberculosis control. In Policy research Working paper WS 4295, volume 1. The World Bank; Human Development Network; Health, Nutrition and Population Team, August 2007. 
[25] S. L. Lenhart and J. T. Workman. Optimal Control Applied to Biological Models. Chapman Hall/CRC, 2007.

[26] M. McAsey, L. Mou, and W. Han. Convergence of the forward-backward sweep method in optimal control. Computational Optimization and Applications, -:1-20, 2012.

[27] D. P. Moualeu, S. Röblitz, R. Ehrig, and P. Deuflhard. Parameter identification for a tuberculosis model in cameroon. Technical Report 13-72, ZIB, Takustr.7, 14195 Berlin, 2013.

[28] D. P. Moualeu-Ngangue. A Mathematical Tuberculosis Model in Cameroon. PhD thesis, 2013.

[29] C. Murray, K. Styblo, and A. Rouillon. Tuberculosis in developing countries: burden, intervention, and cost. Bull. Int. Union Tuberc. Lung Dis., 65:6-24, 1990.

[30] R. L. M. Neilan, E. Schaefer, H. Gaff, K. R. Fister, and S. Lenhart. Modelling optimal control strategies for cholera. Bulletin of Mathematical Biology, Springer New York, 72:2004-2018, 2010.

[31] NIS. Rapport sur la présentation des résultats définitifs. Technical report, National Institute of Statistics, Bureau Central des Recensements et des Etudes de Population, 2010.

[32] P. Nunn, B. G. Williams, K. Floyd, C. Dye, G. Elzinga, and M. C. Raviglione. Tuberculosis control in the era of HIV. Nat. Rev. Immunol., 5:819826, 2005.

[33] D. Okuonghae and V. U. Aihie. Optimal control measures for tuberculosis mathematical models including immigration and isolation of infective. Journal of Biological Systems, 18(01):17-54, 2010.

[34] L. S. Pontryagin, V. G. Boltyanskii, R. V. Gamkrelize, and E. F. Mishchenko. The Mathematical Theory of optimal processes. Wiley, New York, 1962.

[35] S. Russell. The economic burden of illness for households in developing countries: a review of studies focusing on malaria, tuberculosis, and human immunodeficiency virus/acquired immunodeficiency syndrome. Am J Trop Med Hyg, 71(2 suppl):147-55, 2004.

[36] S. P. Sethi. Quantitative guidelines for communicable disease control program: A complete synthesis,. Biometrics, 30(4):681-691, 1974.

[37] C. J. Silva and D. F. M. Torres. Optimal control strategies for tuberculosis treatment: A case study in Angola. Numer. Algebra Control Optim., 2(3):601-617, September 2012. 
[38] WHO. Global tuberculosis control: surveillance, planning, financing. Technical report, World Health Organization, Geneva, Switzerland, 2009.

[39] WHO. Fact sheet number 104. Technical report, World Health Organization, Geneva, Switzerland, 2012.

[40] WHO. Global tuberculosis control: surveillance, planning, financing. Technical report, World Health Organization, Geneva, Switzerland, 2012.

[41] X. Yan, Y. Zou, and J. Li. Optimal quarantine and isolation strategies in epidemics control. World Journal of Modelling and Simulation, 33:202-211, 2007. 